\title{
A Footnote to Medical History: David Alexander Shirres on Spinal Cord Regeneration
}

\author{
Preston Robb
}

Little attention has been paid to an early Canadian experiment in neuronal regeneration and what may have been the world's first attempt to replace a damaged spinal cord with a transplant. In 1905, a paper entitled "Regeneration of the Axones of Spinal Neurones in Man" was published in the Montreal Medical Journal. It had been read at the Panamerican Congress in Panama. The author was David Alexander Shirres, a Scot who had trained in Aberdeen in neurology and neuropathology. He came to Canada in 1902 to assume the position of neurologist at the Montreal General Hospital, with the responsibility of establishing clinics and teaching undergraduates about the mysteries of the nervous system. To my knowledge, he was the first man in Canada to be appointed as a neurologist. (There were others, notably James Stewart, who devoted most of their time and writing to diseases of the nervous system but considered themselves to be internists. Stewart, for example, left the MGH to become the first chief of medicine at the Royal Victoria Hospital in Montreal.)

In his article, after a prolonged discussion of the effects of compression and transsection of the spinal cord, Dr. Shirres described a patient of Dr. G.D. Armstrong who had come under his observation. To set the scene, let us recall that the time was 1902 and the place the old Montreal General Hospital on Lagauchetière Street. Although the antiseptic techniques of Lister were fully established, there were no antibiotics, and surgery would have been done in the old amphitheatre with its tiers of heavily painted seats overlooking the operating site, the view unimpeded by glass.

"J.C., aged 48 years, a sailor, was brought into the Montreal General Hospital, under Dr. Armstrong's care in the spring of 1902, suffering from fracture dislocation of the spinal column in the region of the ninth and tenth dorsal vertebrae, with symptoms of flaccid paralysis, complete loss of motion and sensation and of the superficial rectal retention. Twenty-four hours after admission, Dr. Armstrong cut down over the seat of the fracture and removed the laminae of the ninth and tenth dorsal vertebrae. Upon opening the dura, a complete severance of the cord was found in this situation, with a gap of fully half an inch in extent between the two ends. On stimulation of the anterior roots of the first and second lumbar segments with mild faradic current, with needle electrodes, the patient being only very slightly under the influence of general anaesthetic, contractions took place in the muscles of the leg. Stimulation was then applied to the posterior roots of the same segment and this was also followed by contraction of the muscles.

"At the end of six months, no alteration taking place, I felt justified in holding to Bastian's opinion, that total transverse division of the cord is followed by permanent flaccid paralysis, sensory loss and abolition of the reflexes...

"Eleven months after the operation, and just about the time that the work of Purves Stuart and Ballance was brought out, stimulating our interest in the regeneration of peripheral nerves, Dr. Armstrong wished to know if I could suggest anything in the way of helping our patient... He asked me if I thought it would be of any use to transplant a dog's cord between the ends of the divided cord of our patient. My reply was that I did not know what would occur, but did not think that any serious results would follow and it might be worthy of a trial. He decided to have this operation carried out. A large dog was obtained, place under chloroform, and an operation to expose the cord was carefully done under the most strict asepsis by two assistants, Dr. Barlow and Dr. Campbell. While this was being done our patient was put under chloroform and placed on the table. An incision was made over the site of the old lesion, the dura opened and the cord exposed. At this time we found that a separation now existed between the two ends of the cord, about one and a half inch in extent. With a mild faradic current the anterior and posterior roots in the lower segment of the cord were stimulated, and a faint response took place in the muscles, the dog's cord to the extent of three inches was laid alongside the upper and lower segments of the patient's, a few fine stitches united the pia arachnoid of the one to the other, the dura mater was closed, the wound sutured, a plaster jacket applied and the patient was taken back to the ward. He made a perfect recovery from the operation, the temperature on no occasion going over 100 .

"In the summer of 1903, I had the pleasure of informing Sir Victor Horsely of this operation being carried out, and the 
results above mentioned, and he made the flattering remark that he thought Dr. Armstrong had achieved a great triumph in surgery in having carried out this operation without any septic results following; as one is well aware how difficult it is to operate on a dog with perfect aseptic technique.

"A month passed without any apparent change. Fortunately, I had another group of students who attended my voluntary Christmas Vacation Course. I was able to instill in them an interest in the case, and was thereby able to again obtain help in giving the electricity and massage that I could not otherwise have done. The fifth week after the operation the patient was conscious of flatus in the lower quadrant of the abdomen... Two months after the operation, he described very vividly and with all assurance subjective disturbances in both feet extending up to the knees. The passage of the catheter and the evacuation of the bowels were much more clearly felt.

"At first we were inclined to think that this must be purely imagination, but when one heard the patient describe the condition with such exactitude, its coming and going, one began to think otherwise... Neither at this time or at any time since the accident had voluntary movement or return of objective sensory symptoms taken place. The reflexes, both superficial and deep were still absent.

"Sixteen days after this, for some unaccountable reason, the temperature ran up to 103 degrees. From that day the patient began to lose his appetite and failed physically... At postmortem a large abscess was found in the right kidney...

"The cord was carefully removed and placed in Muller fluid to harden. Six weeks later I opened the dura and found lying between the two ends of the cord, a diffluent mass. Sections of the cord above and below the lesion were put aside for PalWeigert stain... The sections of the upper segment revealed the typical ascending degeneration... The sections below showed definite degeneration in the crossed and direct pyramidal tracts. The dura with its adherent substances which lay between the ends of the cord, showed a mass of minute myelin sheaths of nerve fibres which you may see by looking through the microscope placed before you. These fibres can be seen lying closely adherent to the dura mater and when traced upwards and downwards through the various sections, unite with the segments of the cord above and below, demonstrating the fact that regenera- tion of the axones of the spinal neurons had taken place, to a limited extent...

"I only want to state here the following facts, which you will see demonstrated under the microscope, that the nerve fibres are present and that they unite the segments of the cord."

After reading Shirres' paper over and over again, the reader remains uncertain how long the patient lived after the second operation. Adding up the various times mentioned, we can conclude that he survived three months. Especially intriguing was the use of students to stimulate the muscles after each operation. (Around 1923, Dr. Colin Russel enlisted the help of medical students to provide artificial respiration to a fellow student suffering from Guillain-Barre Syndrome, then called ascending Landry's paralysis.)

Two significant observations emerge from this paper: first, the importance of maintaining activity in denervated muscles until recovery, if any occurs and second, "that regeneration of the axones of the spinal neurones had taken place, to a limited extent".

Patients who have had a loss of function from slow spinal cord compression such as a tumor often have a remarkable recovery after decompression. Patients with a sudden paraplegia from what could be spinal cord concussion may make a complete recovery. Most patients who have suffered a traumatic spinal cord compression, however, do not show any significant recovery. To this day, there is no effective way of restoring function in established traumatic paraplegia or quadriplegia, nor is there likely to be one after the fact. Our greatest hope remains the prevention of motorcycle, automobile, or sporting accidents. Surgical decompression does not change the picture, and it is unrealistic to expect surgical reunion of spinal cord ever to be successful.

Nevertheless, considering the date of the amazing experiment Shirres and Armstrong conducted, one cannot help but be impressed by the audacity of these intrepid physicians. They may have been the first to attempt to replace a damage spinal cord with a transplant, but they certainly were not the last.

\section{REFERENCES}

Shirres, D.A. Regeneration of the spinal neurones in man. Montreal Medical Journal, XXXIV April 1905, 239. 